\title{
Research Article \\ Size Effects of Closed Encounter Ag Nanoshell Pairs for SERS Application
}

\author{
Zhiguo Zhao $\mathbb{D}$, , ${ }^{1,2}$ Wenwen Liu $\left(\mathbb{D},{ }^{1}\right.$ Junfeng Zhao $\left(\mathbb{D},{ }^{1}\right.$ Xiaoyan Dong $\mathbb{D}^{1},{ }^{1}$ Caiqiong Li $(\mathbb{D})$, \\ Pengcheng Zhu $\mathbb{D}^{1},{ }^{1}$ Xinxiang Yu $\left(\mathbb{0},{ }^{1}\right.$ and Han Dai $\mathbb{}^{1}{ }^{1}$ \\ ${ }^{1}$ Laboratory of Advanced Light Alloy Materials and Devices, Postdoctoral Workstation of Nanshan Group Co., Ltd., \\ Yantai Nanshan University, Longkou 265713, China \\ ${ }^{2}$ Hang Xin Material Technology Co. Ltd., Longkou 264006, China
}

Correspondence should be addressed to Han Dai; daihan1985@189.cn

Received 15 December 2020; Revised 10 February 2021; Accepted 15 February 2021; Published 24 February 2021

Academic Editor: Bo Tan

Copyright (c) 2021 Zhiguo Zhao et al. This is an open access article distributed under the Creative Commons Attribution License, which permits unrestricted use, distribution, and reproduction in any medium, provided the original work is properly cited.

\begin{abstract}
Closed encounter Ag nanoshell pairs with remarkable improved plasmonic light enhancement in their gaps have been attracting much attention in the production of sensitivity SERS substrates. This work demonstrates the size effects of Ag nanoshell pairs on obtaining higher light intensity in their gaps. It is found that very complex light intensity changes occur in the gaps of Ag nanoshell pairs with their diameter enlargements (diameters $>100 \mathrm{~nm}$ ). By the calculation of scattering efficiency and electric field vectors, the size-related light intensity changes in the gaps have been revealed and been concluded systematically. This work fills in the gaps of application of nanoshell pairs with larger sizes in SERS detectors and could guide the design of some other Ag nanoshell pair-based optical devices.
\end{abstract}

\section{Introduction}

Noble metallic nanoshells such as $\mathrm{Ag}$ and $\mathrm{Au}$ have been attracting a lot of attention due to their large tunable plasmonic resonance in the visible spectrum [1-3]. Closed encounter $\mathrm{Ag}$ and $\mathrm{Au}$ nanoshells (nanoshell pairs) show more significant light enhancements in their gaps and have remarkable improved plasmonic light enhancement [4], which makes them highly attractive as sensitivity SERS substrates, drug deliverer, etc. [5, 6]. Many experimental and theoretical researches have been focusing on the plasmonic resonance of $\mathrm{Ag}$ and $\mathrm{Au}$ nanoshells with particle diameters smaller than $100 \mathrm{~nm}$ which are used as drug deliverer from the medical safety perspective $[7,8]$. However, few relevant studies have been undertaken on nanoshell pairs with larger diameters.

As reported, the significant light intensity enhancement in the gaps of nanoshell pairs can be attributed to the highly hybridized plasmon modes [9], which are mainly dependent on their distance, shell thickness, core dielectric functions, and diameters [10]. Among these parameters, size- (shell thickness, diameters) induced plasmonic oscillation order changes have significant effects on the light intensity in the gaps of nanoshell pairs. Higher plasmon modes excited by nanoshell pairs with diameters larger than $100 \mathrm{~nm}$ could cause complex response of light intensity in their gaps [11]. Owing to this, more details of size effects are urgently needed to know the nanoshell pair-based SERS devices' design.

The finite difference time domain (FDTD) method which is based on the Max dimensional equation has been widely used to understand the near-field or far-field light of nanomaterials with complex interfaces by calculating the steadystate continuous wave from the time domain signal [12]. Many studies have proved that the FDTD method is an accurate and timesaving way for obtaining the exact optical response of metallic nanostructures $[13,14]$. Therefore, it is adopted herein to reveal the size effects on the light enhancement in the gaps of closed encounter Ag nanoshell pairs. Using FDTD, we found that complex light intensity changes occur in the gaps of Ag nanoshell pairs with the increase in diameters. Through the scattering efficiency and electric field vector calculation, direct relations between the light intensity in the gaps and the plasmonic oscillation have been revealed. 
And based on such relations, various-sized Ag nanoshell pairs with high light intensity in their gaps have been selected for the design of SERS devices.

\section{Materials and Methods}

Generally, low-order oscillation (mainly dipole oscillation) can be supported on Ag nanoparticles and nanoshells with diameter smaller than $100 \mathrm{~nm}$ in the visible spectrum, while only beyond this diameter may higher-order oscillation be excited. Herein, Ag nanoshell pairs with overall diameter $D$ ranging from 50 to $200 \mathrm{~nm}$ and thickness $d$ varying from 5 to $15 \mathrm{~nm}$ have been adopted to study the size effect on the light enhancement in the gaps of Ag nanoshell pairs. The definitions of the diameter $D$ and shell thickness $d$ are presented in Figure 1(a). In our case, the distances between the nanoshell pairs are fixed at a proper value $(10 \mathrm{~nm})$ to exhibit clear changes of the light intensity in the gaps. The absorption and scattering properties of these Ag nanoshell pairs are studied by a plane wave light source with a wavelength ranging from 400 to $800 \mathrm{~nm}$ based on FDTD calculation. To present the size effect on the oscillations, the electric field vectors of $\mathrm{Ag}$ nanoshell pairs with $D=50,100,150 \mathrm{~nm}$, and $200 \mathrm{~nm}$ and $d=7.5 \mathrm{~nm}$ have also been calculated by the FDTD method.

\section{Results and Discussion}

Considering the application of Ag nanoshell pairs in SERS detectors [15], we adopted plane wave sources with a wavelength of $532 \mathrm{~nm}$, the most commonly used wavelength in SERS detectors, to study the size effect on the light intensity changes in the gaps of nanoshell pairs. As shown in Figure 2(a), the light intensity in the gaps of Ag nanoshell pairs with diameters of $50 \mathrm{~nm}$ and a fixed distance of $10 \mathrm{~nm}$ decreased after the thickness was increased from 5 to $15 \mathrm{~nm}$. Nearly contrary changes occur in the gaps of $\mathrm{Ag}$ nanoshell pairs with diameters larger than $50 \mathrm{~nm}$ with the increase in thickness. As shown in Figures 2(b)-2(d), significant light intensity increase can be observed as their thickness increases, especially for $D=200 \mathrm{~nm}$. Ag nanoshell pairs with the greatest thickness obtain the highest light intensity in the gaps, and light enhancement can barely be observed in the gap with a thickness of $5 \mathrm{~nm}$. As a result, the nonlinear relation between the size and the light intensity in their gaps makes it difficult to choose the proper diameters and thickness of Ag nanoshell pairs applied for SERS.

It is known that the scattering/absorption efficiency can directly reflect the plasmonic oscillation excitation of metallic nanostructures [16]. To well know such size-dependent light intensity in the gaps of Ag nanoshell pairs, Figure 3 presents the scattering cross section of Ag nanoshell pairs with $D$ ranging from 50 to $200 \mathrm{~nm}$ and $d$ ranging from 5 to $15 \mathrm{~nm}$ across the entire visible spectrum. Clear scattering peaks of Ag nanoshell pairs can be observed in Figure 3(a), in which the peak shifts from $405 \mathrm{~nm}$ to $540 \mathrm{~nm}$ as $d$ decreased from 15 to $5 \mathrm{~nm}$. And slight peak broadening can also be observed. Such phenomenon can be explained by the hybridization theory [17], which states that the hybridization of oscillation of both cavity and nanoparticles causes the bonding $\omega_{+}$and

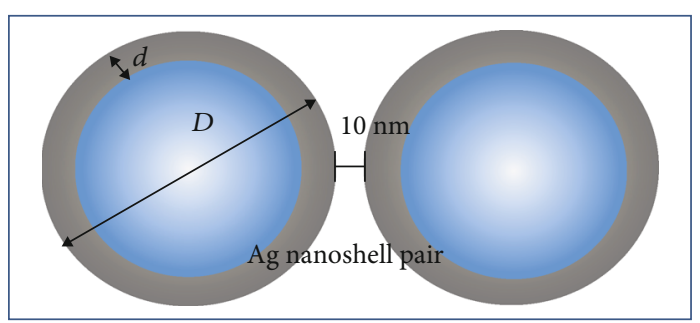

Figure 1: Schematic diagram of the nanoshell pairs.

antibonding $\omega_{\text {- }}$ hybridized plasmon modes. The detailed explanation can be concluded as

$\omega_{ \pm}=\omega_{\mathrm{B}}^{2}\left[0.5 \pm \frac{1}{2(2 l+1)} \sqrt{1+4 l(l+1)\left(\frac{D-2 d}{D}\right)^{2 l+1}}\right](l=1,2 \cdots)$,

where $l$ is the angular quantum number, $\omega_{\mathrm{B}}=\sqrt{4 \pi e^{2} n_{0} / m_{\mathrm{e}}}$ is the intrinsic resonance of Ag, and $n_{0}$ and $m_{\mathrm{e}}$ are the free electron density and electron mass, respectively. When $d$ decreases, the bonding plasmon mode $\omega_{+}$increases and the

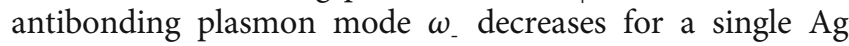
nanoshell causing the hybridization increase, which generally lead to significant red shifts of oscillation peaks. However, $D$ shows the contrary effects with that of $d$. When $D$ decreases, the bonding plasmon mode $\omega_{+}$decreases and the antibond-

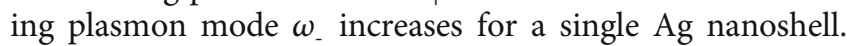
When Ag nanoshells combine into pairs, surface coupling in the gaps between nanoshells could lead to further red shift and peak broadening due to the damping effect [18]. Owing to this, the decrease in $d$ of Ag nanoshell pairs with $D=50$ $\mathrm{nm}$ causes significant red shift and peak broadening in Figure 3(a). And the significant light intensity enhancement in the gap of Ag nanoshell pair with $d=5 \mathrm{~nm}$ in Figure 2(a) can be attributed to such oscillation peak red shift to the wavelength $550 \mathrm{~nm}$, which is quite close to the wavelength of the SERS light source. When $D$ is increased to $100 \mathrm{~nm}$, two oscillation peaks can be clearly observed, as shown in Figure 3(b). Apparently, Ag nanoshell pair with the largest thickness $d=15 \mathrm{~nm}$ has the oscillation peak closest to $532 \mathrm{~nm}$, which shows significant different tendency from that of Ag nanoshell pair with $D=50 \mathrm{~nm}$, which leads to the obvious light intensity enhancement of Ag nanoshell pair as the thickness increase. As shown in Figure 3(c), all of the oscillation peaks are far away from $532 \mathrm{~nm}$, which corresponds to the overall low light intensity with different thicknesses in Figure 2(c). Above all, the nonlinear relation between the size and the light intensity within the gaps of Ag nanoshell pairs can be well explained below: the oscillations peaks of $\mathrm{Ag}$ nanoshell pairs (diameter $50 \mathrm{~nm}$ ) mainly localized to the left side of $532 \mathrm{~nm}$, which is contrary to that of Ag nanoshell pairs (diameters $100 \mathrm{~nm}$ and $200 \mathrm{~nm}$ ). The shell thickness decrease causes the oscillation peak red shift surrounding $532 \mathrm{~nm}$ of Ag nanoshell pairs with a diameter of $50 \mathrm{~nm}$. But for Ag nanoshell pairs with larger diameters $(100 \mathrm{~nm}$ and $200 \mathrm{~nm}$ ), the shell thickness decrease leads to the oscillation 


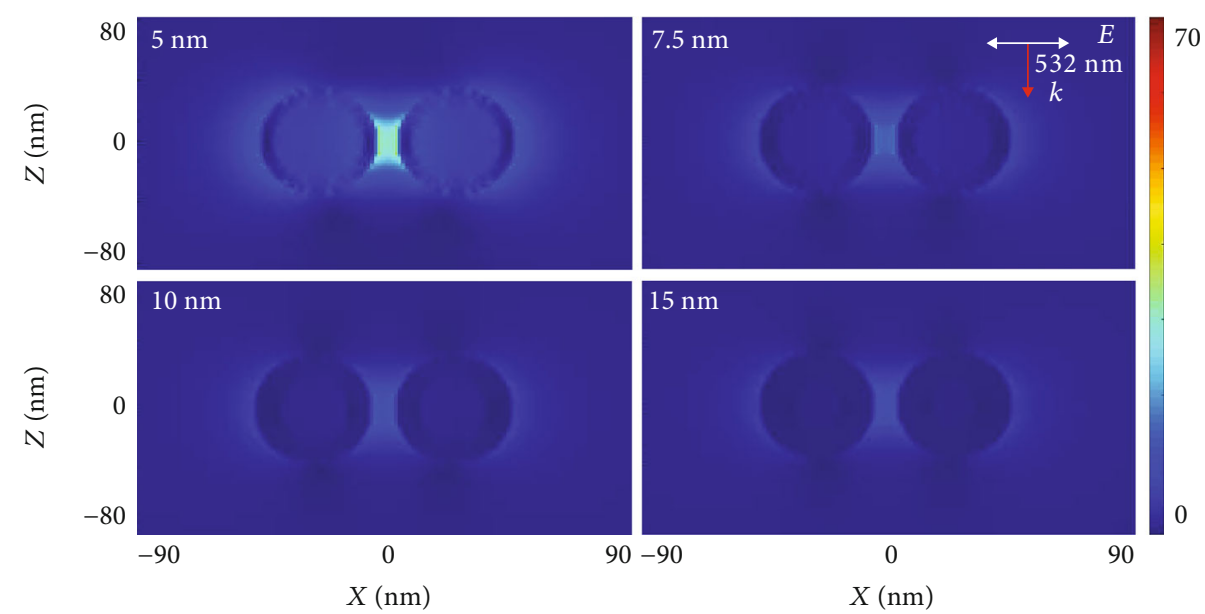

(a)
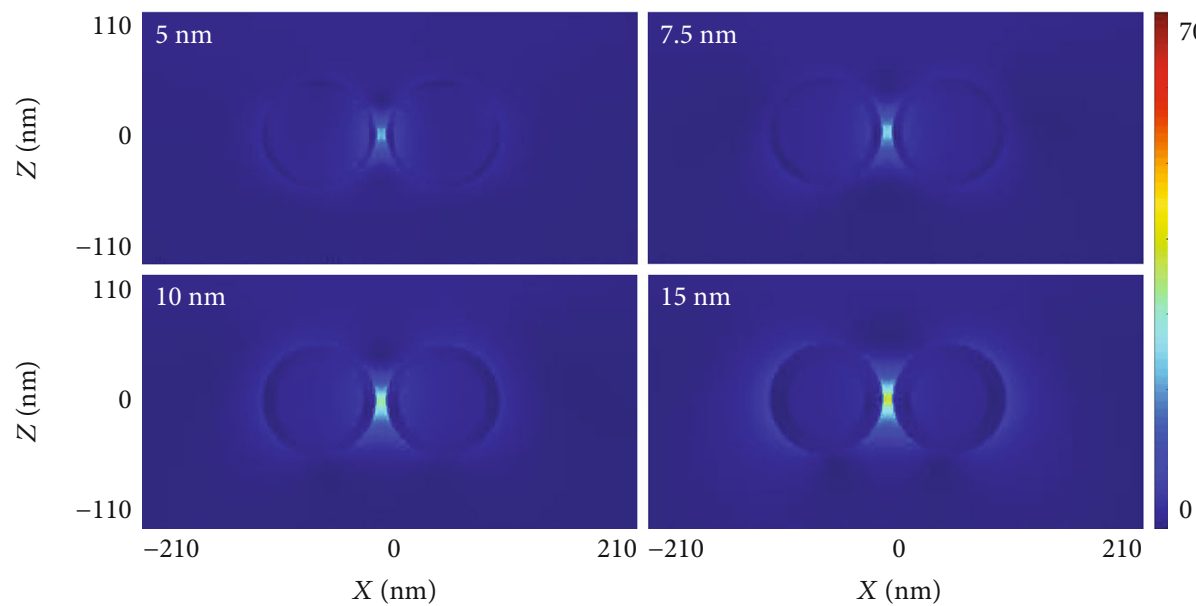

(b)

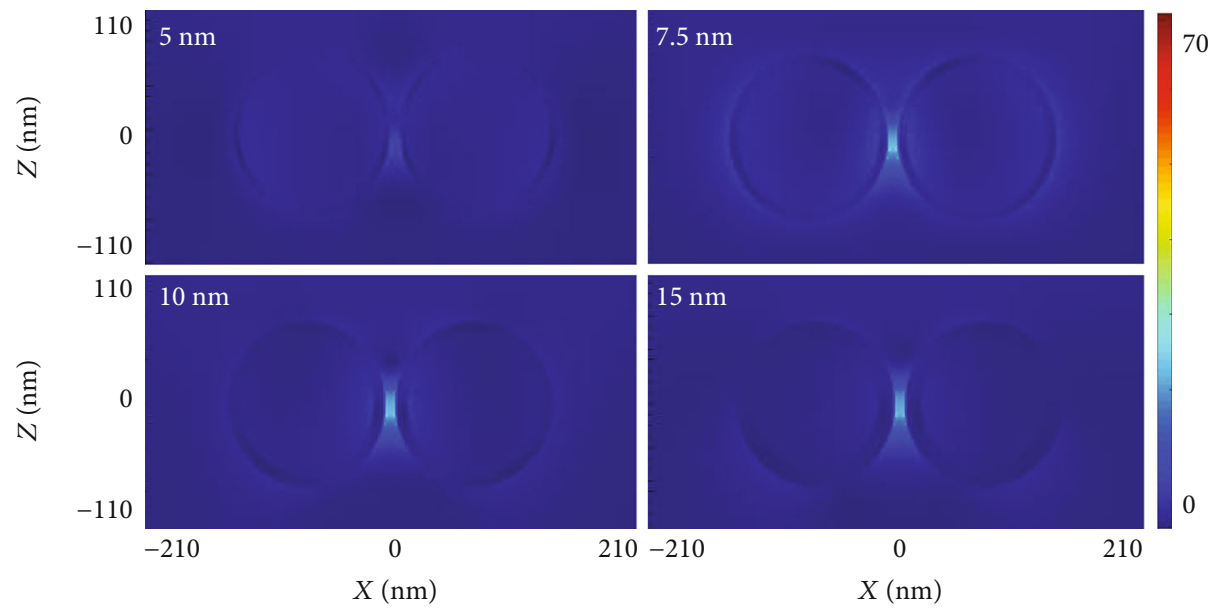

(c)

FIgUre 2: Continued. 


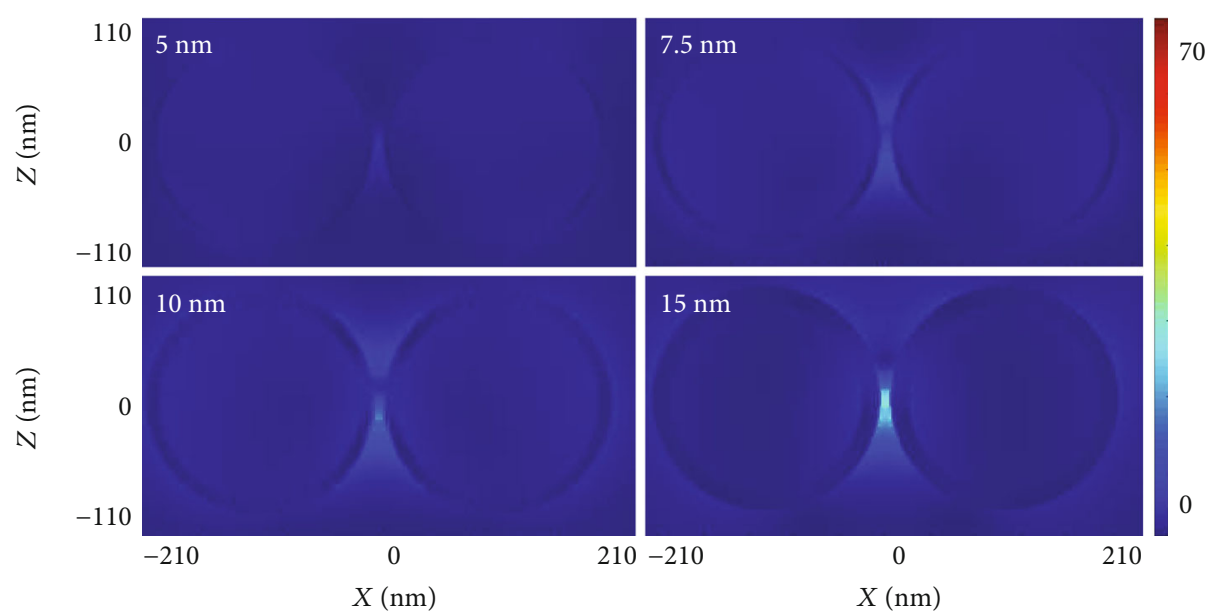

(d)

FIGURE 2: Light intensity in the gaps of Ag nanoshell pairs with various diameters and thickness: (a-d) Ag nanoshell pairs with diameters 50, 100,150 , and $200 \mathrm{~nm}$ and with shell thickness 5, 7.5, 10, and $15 \mathrm{~nm}$.

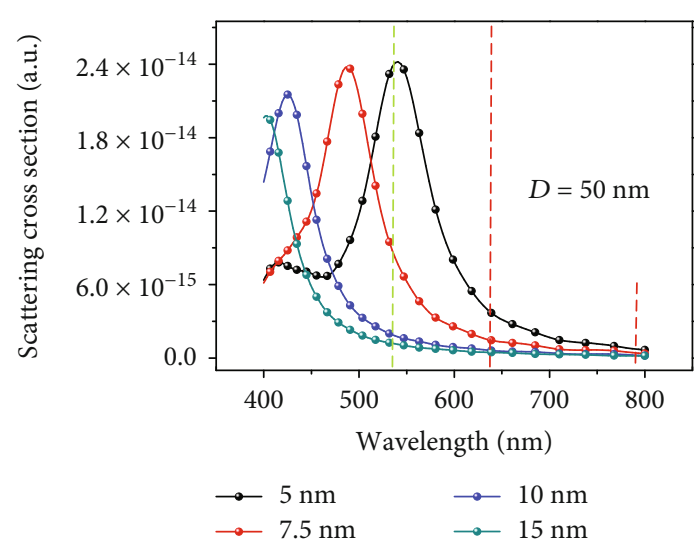

(a)

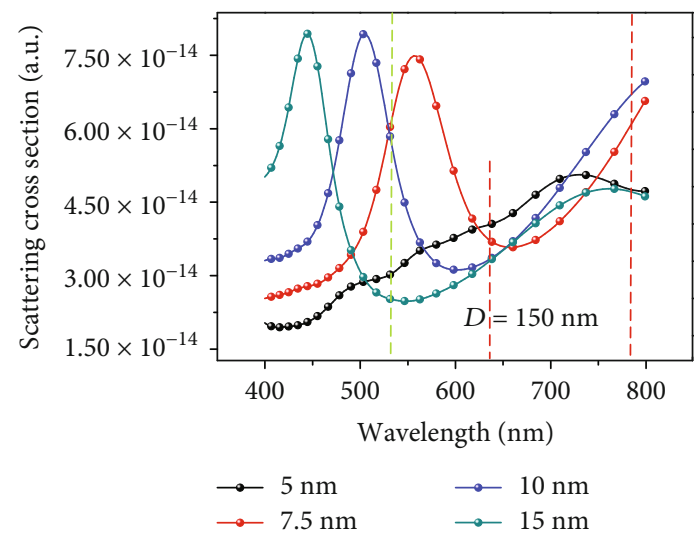

(c)

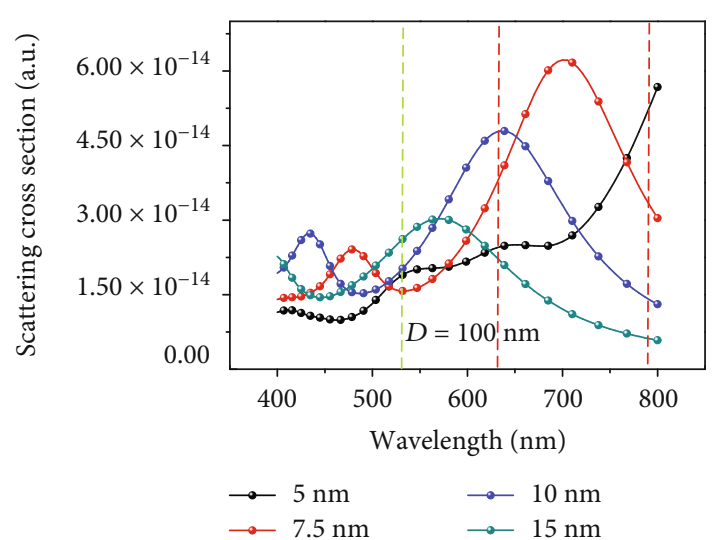

(b)

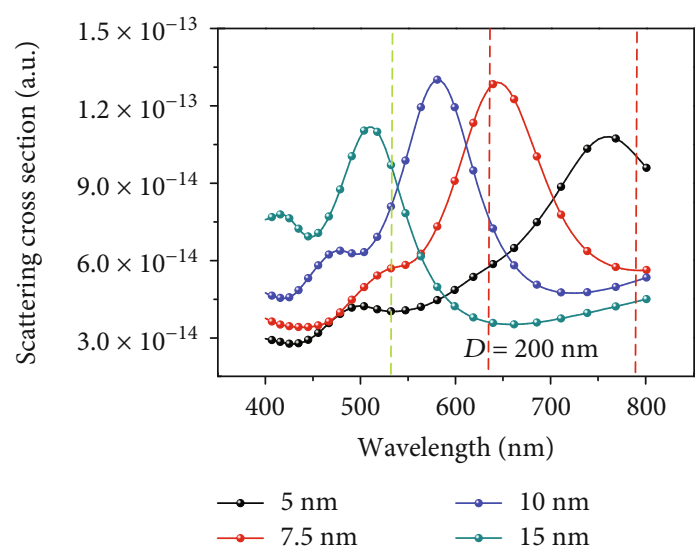

(d)

FIGURE 3: Scattering cross section of Ag nanoshell pairs with various diameters and thickness: (a-d) Ag nanoshell pairs with diameters 50, 100,150 , and $200 \mathrm{~nm}$ and with shell thickness 5, 7.5, 10, and $15 \mathrm{~nm}$. Three common wavelengths (532, 633, and $785 \mathrm{~nm}$ ) of laser sources applied in SERS detectors are marked by dashed lines.

peak red shift far way from $532 \mathrm{~nm}$, which leads to the light intensity decrease within the gaps of $\mathrm{Ag}$ nanoshell pairs. In addition, abnormal blue shifts and peak narrowing of $\mathrm{Ag}$ nanoshell pairs with $D=150 \mathrm{~nm}$ and $d$ increase from 7.5 to
$15 \mathrm{~nm}$ can also be found. However, overall red shifts and peak broadenings can be observed in Figure 3(d) when $D$ increases to $200 \mathrm{~nm}$. Therefore, when selecting the nanoshell pairs with appropriate size, it is necessary to keep them as 


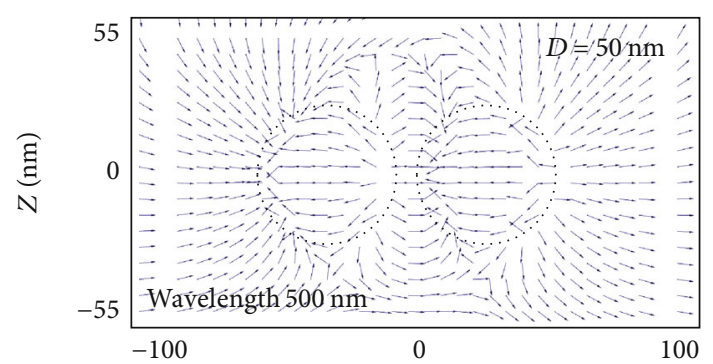

(a)

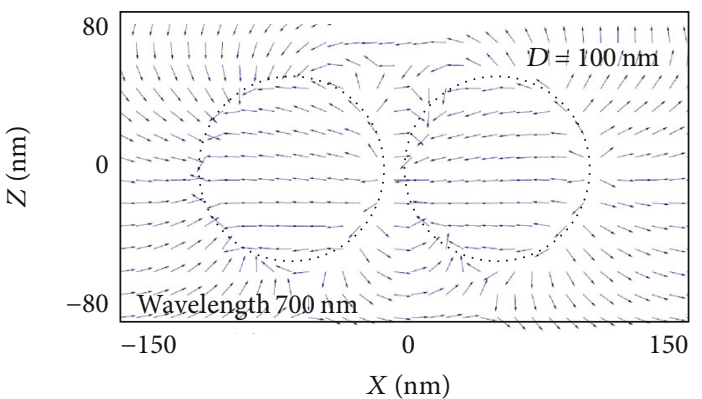

(c)

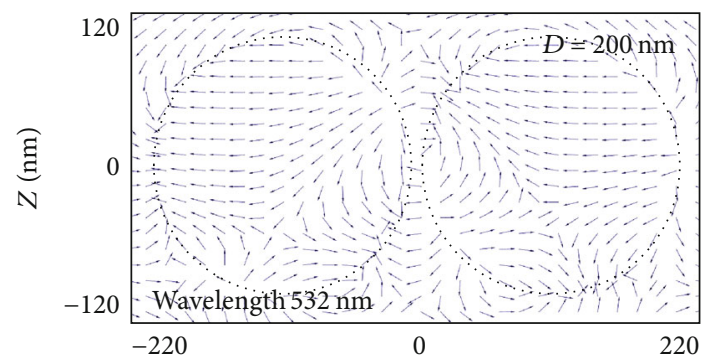

(e)

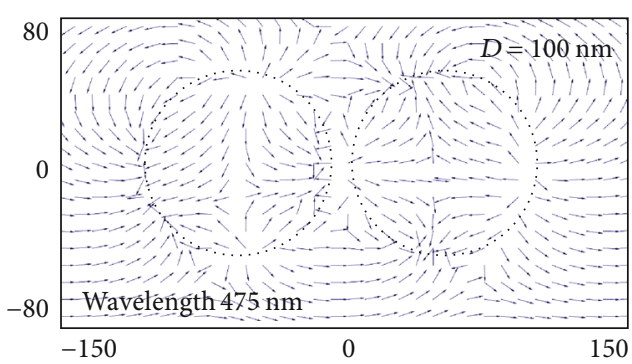

(b)

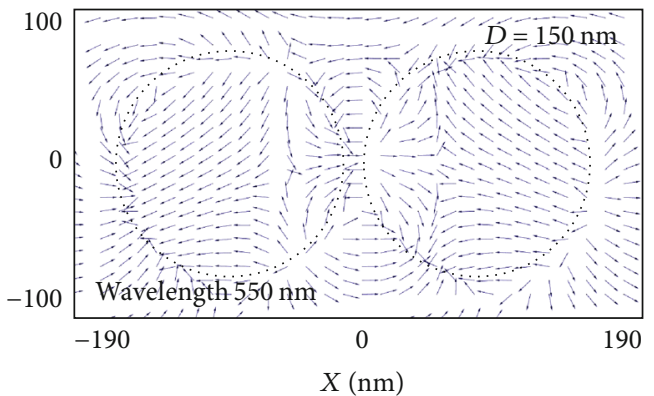

(d)

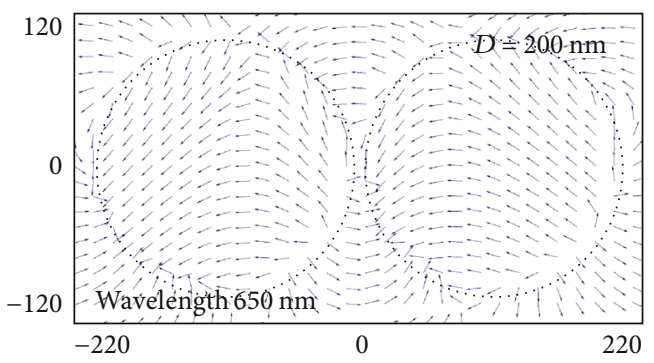

(f)

Figure 4: (a-f) Electric field vector diagram of Ag nanoshell pairs with diameters 50, 100, 150, $200 \mathrm{~nm}$ and thicknesses $7.5 \mathrm{~nm}$.

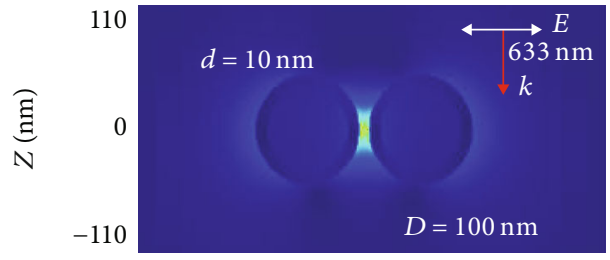

(a)

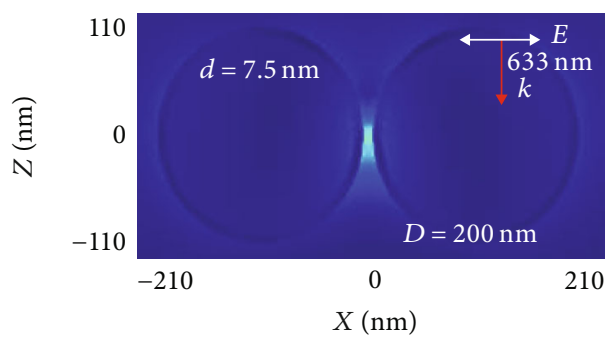

(c)

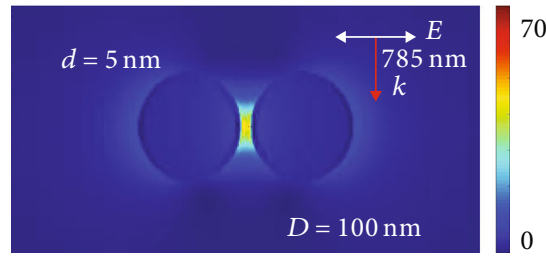

(b)

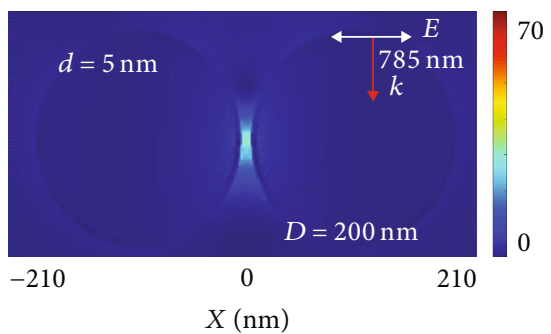

(d)

FIGURE 5: Ag nanoshell pairs with proper sizes to obtain significant light enhancements between their gaps: (a) diameter $100 \mathrm{~nm}$, thickness $10 \mathrm{~nm}$ by $633 \mathrm{~nm}$ light illumination; (b) diameter $100 \mathrm{~nm}$, thickness $5 \mathrm{~nm}$ by $785 \mathrm{~nm}$ light illumination; (c) diameter $200 \mathrm{~nm}$, thickness $7.5 \mathrm{~nm}$ by $633 \mathrm{~nm}$ light illumination; (d) diameter $200 \mathrm{~nm}$, thickness $5 \mathrm{~nm}$ by $785 \mathrm{~nm}$ light illumination. 
close to the oscillation peaks as possible. For better choices, three common wavelengths $(532,633$, and $785 \mathrm{~nm})$ of laser sources applied in SERS detectors are marked with dashed lines in each figures.

To further understand the size effect on the oscillations in Figure 3, especially for explaining the occurrence of the totally abnormal blue shift and peak narrowing with $D=150$ and $200 \mathrm{~nm}$ in Figures 3(c) and 3(d), typical electric field vector diagrams of Ag nanoshell pairs are presented in Figure 4. Figure 4(a) shows obviously the dipole oscillation excited on Ag nanoshell pairs with $D=50 \mathrm{~nm}$, while in Figures 4(b) and $4(\mathrm{c})$ with $D=100 \mathrm{~nm}$, the dipole oscillation corresponds to the $700 \mathrm{~nm}$ peak and the quadrupole oscillation corresponds to $475 \mathrm{~nm}$. Apparently, the phenomenon found in Figure 3(c) is not blue shift and peak narrowing of the dipole oscillation peaks. It could be attributed to the asymmetric quadrupole oscillation, which is enhanced around 450 to $580 \mathrm{~nm}$, as shown in Figure 4(d). For Ag nanoshell pairs with a diameter of $200 \mathrm{~nm}$, more complex higher-order oscillation modes are excited, as shown in Figures 4(e) and 4(f). Obviously, these complex order excitations on Ag nanoshell pairs with large diameters lead to the nonlinear light intensity changes in their gaps. However, whether high-order or loworder oscillation, it is the oscillation efficiencies (mainly oscillation peaks) that determine their light intensity in the gaps.

Considering the potential application of $\mathrm{Ag}$ nanoshell pairs in SERS, the wavelengths of light sources are fixed at $633 \mathrm{~nm}$ and $785 \mathrm{~nm}[19,20]$. In order to obtain sufficient light intensity, four models of Ag nanoshell pairs with proper sizes have been adopted, as shown in Figure 5. Comparison among these four figures has been conducted, and it was found that nanoshell pairs with $D=100 \mathrm{~nm}$ and $d=5 \mathrm{~nm}$ by $785 \mathrm{~nm}$ light illumination show the highest light intensity (Figure 5(b)) in the gaps, which should be attributed to the significant oscillation peak around $850 \mathrm{~nm}$. Apparently, the oscillation peak of the Ag nanoshell pair has such size only close to the wavelength of light sources but simultaneously has significant high value. It can be seen clearly that the others which are close to the oscillation peak also have sufficiently high light intensity in their gaps, as shown in Figures 5(a), 5(c) and 5(d). Obviously, the light intensity can be easily tuned to meet SERS detection requirements with various light sources simply by choosing appropriate sizes.

\section{Conclusions}

In summary, the size effects of Ag nanoshell pairs on their light enhancement between their gaps have been demonstrated by the FDTD method. Firstly, the light intensity in the gaps of $\mathrm{Ag}$ nanoshell pairs with various $D$ and $d$ at a wavelength of $532 \mathrm{~nm}$ was calculated. Due to the high-order oscillation excited on Ag nanoshell pairs with $D$ larger than $50 \mathrm{~nm}$, complex tendencies of the light intensity changes in the gaps have been observed. Through the calculation of the scattering efficiency and electric field vectors of Ag nanoshell pairs, the relations between the size of Ag nanoshell pairs and the light intensity in their gaps have been concluded systematically. In addition, the size change induced oscillation peak shift and peak broadening/narrowing has also been presented. Furthermore, by adopting the appropriate sizes, some typical models of Ag nanoshell pairs with high light intensity have also been provided. This work not only offers the theoretical basis for the design of nanoshell-based SERS detectors but could also benefit some other Ag nanoshell pair-based optical devices.

\section{Data Availability}

The data used to support the findings of this study are included within the article.

\section{Conflicts of Interest}

The authors declare that there is no conflict of interest regarding the publication of this paper.

\section{Authors' Contributions}

Zhiguo Zhao and Wenwen Liu contributed equally to this work.

\section{Acknowledgments}

We thank Nanshan Holdings for the materials and equipment support. This research study was supported by Yantai Double Hundred Talent Plan 2019 and Postdoctoral Innovation Project of Shandong Province in 2020. This research was funded by China Postdoctoral Science Foundation, Grant No. 2020M671987; Shandong Provincial Natural Science Foundation, China Grant No. ZR2020ME005; Yantai University and Local Integration Project, China, Grant No. 2020XDRHXMPT18; Longkou science and technology research and development plan project, Grant No. 2019KJJH021; and Doctoral Fund of Yantai Nanshan University, China, Grant Nos. B202002, B202003, B202006, Q202013, and Q202015.

\section{References}

[1] S. S. Kim, S. I. Na, J. Jo, D. Y. Kim, and Y. C. Nah, "Plasmon enhanced performance of organic solar cells using electrodeposited Ag nanoparticles," Applied Physics Letters, vol. 93, no. 7, p. 305, 2008.

[2] K. C. Lee, S. J. Lin, C. H. Lin, C. S. Tsai, and Y. J. Lu, "Size effect of Ag nanoparticles on surface plasmon resonance," Surface and Coatings Technology, vol. 202, no. 22-23, pp. 5339-5342, 2008.

[3] S. A. Maier, P. G. Kik, and H. A. Atwater, "Observation of coupled plasmon-polariton modes in Au nanoparticle chain waveguides of different lengths: estimation of waveguide loss," Applied Physics Letters, vol. 81, no. 9, pp. 1714-1716, 2002.

[4] W. B. Zou, J. Zhou, L. Jin, and H. P. Zhang, "Properties of localized surface plasmon resonance of gold nanoshell pairs," Acta Physica Sinica, vol. 61, no. 9, article 097805, 2012.

[5] L. R. Hirsch, J. B. Jackson, A. Lee, N. J. Halas, and J. L. West, “A whole blood immunoassay using gold nanoshells," Analytical Chemistry, vol. 75, no. 10, pp. 2377-2381, 2003.

[6] H. N. Xie, I. A. Larmour, W. E. Smith, K. Faulds, and D. Graham, "Surface-enhanced Raman scattering 
investigation of hollow gold nanospheres," The Journal of Physical Chemistry C, vol. 116, no. 14, pp. 8338-8342, 2012.

[7] H. P. Liang, L. J. Wan, C. L. Bai, and L. Jiang, "Gold hollow nanospheres: tunable surface plasmon resonance controlled by interior-cavity sizes," The Journal of Physical Chemistry B, vol. 109, no. 16, pp. 7795-7800, 2005.

[8] J. Z. Zhang, "Biomedical applications of shape-controlled plasmonic nanostructures: a case study of hollow gold nanospheres for photothermal ablation therapy of cancer," The Journal of Physical Chemistry Letters, vol. 1, no. 4, pp. 686695, 2010.

[9] E. Prodan, C. Radloff, N. J. Halas, and P. Nordlander, “A hybridization model for the plasmon response of complex nanostructures," Science, vol. 302, no. 5644, pp. 419-422, 2003.

[10] K. L. Kelly, E. Coronado, L. L. Zhao, and G. C. Schatz, The optical properties of metal nanoparticles: the influence of size, shape, and dielectric environment, 2003.

[11] D. W. Brandl, C. Oubre, and P. Nordlander, "Plasmon hybridization in nanoshell dimers," The Journal of Chemical Physics, vol. 123, no. 2, article 024701, 2005.

[12] W. M. Saj, "FDTD simulations of 2D plasmon waveguide on silver nanorods in hexagonal lattice," Optics Express, vol. 13, no. 13, pp. 4818-4827, 2005.

[13] G. Y. Yao, Q. L. Liu, and Z. Y. Zhao, "Studied localized surface plasmon resonance effects of au nanoparticles on $\mathrm{TiO}_{2}$ by FDTD simulations," Catalysts, vol. 8, no. 6, p. 236, 2018.

[14] C. Tira, D. Tira, T. Simon, and S. Astilean, "Finite-difference time-domain (FDTD) design of gold nanoparticle chains with specific surface plasmon resonance," Journal of Molecular Structure, vol. 1072, pp. 137-143, 2014.

[15] J. A. Dougan, D. Mac Rae, D. Graham, and K. Faulds, "DNA detection using enzymatic signal production and SERS," Chemical Communications, vol. 47, no. 16, pp. 4649-4651, 2011.

[16] W. Ni, X. Kou, Z. Yang, and J. Wang, "Tailoring longitudinal surface plasmon wavelengths, scattering and absorption cross sections of gold nanorods," ACS Nano, vol. 2, no. 4, pp. 677686, 2008.

[17] E. Prodan and P. J. C. P. Nordlander, "Plasmon hybridization in spherical nanoparticles," The Journal of Chemical Physics, vol. 120, no. 11, pp. 5444-5454, 2004.

[18] F. Peyskens, A. Z. Subramanian, P. Neutens et al., "Bright and dark plasmon resonances of nanoplasmonic antennas evanescently coupled with a silicon nitride waveguide," Optics Express, vol. 23, no. 3, pp. 3088-3101, 2015.

[19] Q. Su, X. Ma, J. Dong, C. Jiang, and W. Qian, “A reproducible SERS substrate based on electrostatically assisted APTESfunctionalized surface-assembly of gold nanostars," ACS Applied Materials \& Interfaces, vol. 3, no. 6, pp. 1873-1879, 2011.

[20] C. Muehlethaler, K. Ng, L. Gueissaz, M. Leona, and J. R. Lombardi, "Raman and SERS characterization of solvent dyes: an example of shoe polish analysis," Dyes and Pigments, vol. 137, pp. 539-552, 2017. 